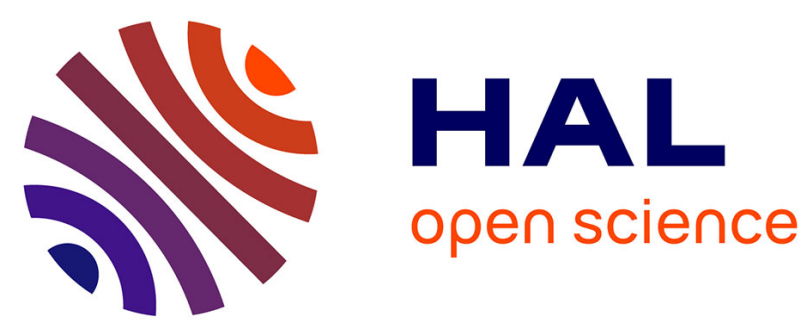

\title{
Épidémiologie de l'artériopathie des membres inférieurs
}

\author{
Victor Aboyans, Marie-Antoinette Sevestre, Iléana Desormais, Philippe
}

Lacroix, Gerry Fowkes, Michael Criqui

\section{To cite this version:}

Victor Aboyans, Marie-Antoinette Sevestre, Iléana Desormais, Philippe Lacroix, Gerry Fowkes, et al.. Épidémiologie de l'artériopathie des membres inférieurs. La Presse Médicale, 2018, 47 (1), pp.38-46. 10.1016/j.lpm.2018.01.012 . hal-02042922

\section{HAL Id: hal-02042922 \\ https://hal-unilim.archives-ouvertes.fr/hal-02042922}

Submitted on 3 Sep 2020

HAL is a multi-disciplinary open access archive for the deposit and dissemination of scientific research documents, whether they are published or not. The documents may come from teaching and research institutions in France or abroad, or from public or private research centers.
L'archive ouverte pluridisciplinaire HAL, est destinée au dépôt et à la diffusion de documents scientifiques de niveau recherche, publiés ou non, émanant des établissements d'enseignement et de recherche français ou étrangers, des laboratoires publics ou privés. 


\title{
Épidémiologie de l'artériopathie des membres inférieurs
}

\author{
Victor Aboyans ${ }^{1,2}$, Marie-Antoinette Sevestre ${ }^{3}$, Ileana Désormais ${ }^{2,4}$, Philippe Lacroix ${ }^{2,4}$, Gerry Fowkes \\ ${ }^{5}$, Michael H. Criqui ${ }^{6}$ \\ 1. CHU de Limoges, service de cardiologie, 87042 Limoges, France \\ 2. Faculté de médecine, Inserm U1094, 87025 Limoges, France \\ 3. CHU d'Amiens, service de médecine vasculaire, 80054 Amiens, France \\ 4. CHU de Limoges, service de chirurgie thoracique et vasculaire et médecine \\ vasculaire, 87042 Limoges, France \\ 5. Usher institute of population health sciences and informatics, Edimbourg, \\ Royaume-Uni \\ 6. University of California, department of family medicine and public health, San \\ Diego, La Jolla, CA, États-Unis \\ Correspondance : \\ Victor Aboyans, CHU Dupuytren de Limoges, service de cardiologie, 2, \\ avenue Martin-Luther-King, 87042 Limoges cedex, France. \\ victor.aboyans@unilim.fr
}

\section{Résumé}

On estime à plus de 200 millions le nombre d'individus touchés par l'artériopathie des membres (AOMI) dans le monde. Cette prévalence aurait augmenté d'environ $25 \%$ entre 2000 et 2010, et notamment dans les pays à revenu faible/intermédiaire. En France, on estime que près d'un million de français présenterait cette maladie. Près de deux-tiers de la population atteinte d'AOMI présente sa forme asymptomatique. Ainsi, la majorité des études épidémiologiques ont recours à la mesure de l'index de pression systolique (IPS). Un IPS 0,90 signe la présence d'une AOMI. La détection des formes symptomatiques de l'AOMI nécessite le recours à des questionnaires standardisés, permettant de diagnostiquer la claudication intermittente. Les études concernant l'ischémie critique - la forme la plus sévère de la maladie - sont encore rares : on estime dans les pays occidentaux une prévalence de l'ordre de $0,5-2,0 \%$ dans la population de plus de 40 ans. Comme toute maladie athéromateuse, les facteurs de risque sont de 4 ordres (facteurs génétiques, les facteurs de risque traditionnels, les facteurs métaboliques et inflammatoires, et les facteurs psycho-socioéconomiques) avec néanmoins des poids différents par rapport à la maladie coronaire. Du fait de leur grande prévalence et leur association avec la survenue de l'AOMI, le tabagisme et l'hypertension artérielle sont les deux premiers pourvoyeurs de cette maladie en population. Le diabète est un puissant facteur de risque, et son essor dans la population contribue à l'augmentation de la prévalence de l'AOMI. Chez le claudicant, le risque d'amputation est de $5 \%$ à 5 ans, passant à $25 \%$ à un an en cas d'ischémie critique. Mais le risque principal est celui des évènements cardiovasculaires tels que l'infarctus et l'accident vasculaire cérébral. On estime que $50 \%$ de ces patients ont une atteinte coronaire concomitante, et $20 \%$ ont une atteinte cérébrovasculaire. La mortalité non cardiovasculaire, essentiellement liée au cancer, est également plus importante que la population générale. Globa-lement, le contrôle des facteurs de risque traditionnels a un effet favorable tant sur la diminution du risque de survenue de la maladie que son évolution et ses complications. 


\section{Summary}

Epidemiology of lower extremity artery disease

It is estimated that more than 200 million individuals are affected by lower extremity artery disease (LEAD) worldwide. This prevalence has increased between 2000 and 2010 by $25 \%$, especially in low/middle income countries. In France, about one million people are affected by this condition. Almost two-thirds of patients with LEAD are asymptomatic. This explains the interest of the measurement of the ankle-brachial index (ABI), an objective and harmless diagnostic tool. An ABI $\square 0.90$ is considered as diagnostic for LEAD. The detection of symptomatic LEAD requires standardized questionnaires identifying intermittent claudication. Epidemiological studies on chronic limb-threatening ischemia (CLTI) - the most severe presentation of the disease are scarce: the prevalence is estimated around $0.5-2.0 \%$ after the age of 40 , mostly affecting elderly people. Similar to other atherosclerotic diseases, the risk factors are multiple (genetic factors, traditional risk factors, metabolic and inflammatory factors, socioeconomic factors), with different weighs of association as compared to coronary artery diseases. Due to their high prevalence and strength of association, cigarette smoking and hypertension are the most frequent purveyors of this disease in population. Diabetes mellitus is a strong risk factor, and its increasing prevalence contributes to the global epidemics of LEAD. In claudicants, the 5 -year amputation risk is estimated at $5 \%$, increasing to $25 \%$ at one year in case of CLTI. However, the main risk is related to general cardiovascular events. It is estimated that patients with LEAD present concomitantly coronary and cerebrovascular disease in respectively $50 \%$ and $20 \%$ of cases. The non-cardiovascular mortality, especially related to cancer, is also higher than in general population. Overall, the control of traditional risk factors has a beneficial effect both for the limb and general prognosis.

artériopathie oblitérante des membres inférieurs (AOMI) est un des principaux modes d'expression de la maladie athéromateuse, affectant l'arbre artériel de la terminaison de l'aorte jusqu'aux artères du pied. Du fait de leur rareté, les atteintes non athéromateuses n'ont jamais spécifiquement fait objet d'étude épidémiologique.

La plupart des données épidémiologiques sont issues d'études réalisées dans les pays riches, mais ces dernières années plusieurs études dans les pays en voie de développement montrent également la grande fréquence de cette maladie dans ces populations [1]. On estime à plus de 200 millions le nombre d'individus touchés par cette maladie dans le monde [1]. D'après les mêmes estimations, cette prévalence aurait augmenté d'environ $25 \%$ entre 2000 et 2010 [1].

\section{Définition épidémiologique de l'AOMI}

L'AOMI présente habituellement une longue période de développement infraclinique, avant la présentation de symptômes, débutant généralement par l'apparition de douleurs des membres à l'effort, dont l'expression typique est la claudication intermittente $(\mathrm{Cl})$. Dans sa forme la plus grave, l'ischémie critique se présente par des douleurs au repos et/ou des lésions tissulaires à type d'ulcères artériels et de nécrose. Près de deuxtiers de la population atteinte d'AOMI présente sa forme asymptomatique $[2,3]$. Si les premières études épidémiologiques dans ce domaine se sont attachées à détecter la claudication intermittente, la plupart des études de ces 30 dernières années ont étudié cette maladie en faisant le diagnostic par une méthode simple et objective, pouvant également détecter la forme asymptomatique, par la mesure de l'index de pression systolique (IPS) $[2,3]$. Cette méthode présente d'autant plus intérêt depuis qu'il a été démontré, que même dans sa forme asymptomatique, l'AOMI est associée à un risque augmenté d'accident cardiovasculaire $[4,5]$.

L'IPS est le rapport de pression systolique de cheville par celui du bras. Sa mesure a été standardisée par un document de consensus [6]. L'intérêt principal de cette méthode diagnostique est sa large faisabilité, son caractère non invasif, et son faible coût, largement adapté aux études épidémiologiques. Un IPS $\leq 0,90$ est considéré comme anormal et signe la présence d'une AOMI, avec une spécificité de $97 \%$ et une sensibilité de $80 \%$ en population générale [7]. Dans les études de population, I'IPS semble physiologiquement un peu plus faible, de l'ordre de $-0,02$, chez les femmes, et chez les sujets d'origine africaine [8]. Si à l'échelle individuelle et clinique, une telle différence n'a pas de conséquence significative dans la prise en charge des patients, cette différence (cumulée chez les femmes d'origine africaine) peut surestimer la prévalence de l'AOMI dans ces 
populations. On estime que cette différence peut surestimer la prévalence de l'AOMI de $10 \%$ chez les femmes [8].

Il est important à souligner que si le seuil de 0,90 est généralement admis pour définir l'AOMI en épidémiologie, les individus ayant un IPS entre 0,91 et 1,00 sont considérés dans une zone limite, et nombre de ces patients peuvent présenter une chute des pressions de cheville à l'effort, signant authentiquement la maladie [6]. Par ailleurs, certains individus âgés ou diabétiques et/ou insuffisants rénaux (notamment les dialysés) peuvent présenter des IPS très élevés, > 1,40, liée à la médiacalcose. Cette entité correspond à la calcification la couche médiale des artères, rendant celles-ci progressivement incompressibles lors de l'inflation du brassard pour la mesure de l'IPS. La médiacalcose est une pathologie distincte de l'athérosclérose, mais peut y être associée, expliquant pourquoi les patients ayant un IPS > 1,40 peuvent aussi être atteints d'AOMI dans bon nombre de cas, nécessitant des explorations supplémentaires pour l'identifier [6].

La détection des formes symptomatiques de l'AOMI nécessite le recours à des questionnaires standardisés, permettant de diagnostiquer la claudication intermittente, soit de manière certaine, soit considérée comme probable. Le questionnaire de claudication de San Diego, et celui d'Edimbourg sont les plus usités, ce dernier étant validé en version française [9]. Si ces questionnaires manquent de sensibilité et ne sont positifs que dans un sous-groupe de l'ensemble des artériopathes, leur utilisation en épidémiologie permet d'identifier et évaluer les conséquences cliniques notamment sur la qualité de vie de ces patients. Cependant nombre de patients symptomatiques ne présentent pas la forme typique de la claudication, soit parce qu'ils présentent d'autres comorbidités pouvant elles aussi être sources de douleurs des membres inférieurs (par exemple arthropathies), soit parce que la sensibilité nociceptive de ces patients est perturbée (par exemple par une neuropathie diabétique), soit encore parce que les patients atteints, souvent fragiles, ne marchent pas suffisamment pour atteindre le seuil de claudication (par exemple du fait d'une insuffisance cardiorespiratoire associée). Enfin, ces manifestations cliniques peuvent également être perturbées par un état dépressif qui est fréquent chez ces patients [10].

\section{Prévalence}

Une revue systématique récente a permis d'établir la prévalence de l'AOMI, basée sur l'IPS < 0,90, dans les différentes régions du monde, en comparant cette prévalence dans les pays à haut revenu par rapport aux pays à revenu faible/intermédiaire (figure 1) [1]. Rare avant l'âge de 50 ans, la prévalence de I'AOMI augmente de manière exponentielle à partir de 60 ans pour atteindre les $20 \%$ après 80 ans.

Dans les pays à haut revenu, la prévalence est semblable chez les femmes et les hommes. Cette prévalence est de l'ordre de $5 \%$ à l'âge de $45-49$ ans et augmente jusqu'à près de $18 \%$ après 85 ans. On estime à près de 40 millions le nombre d'individus atteints par toute forme d'AOMI sur l'ensemble du continent européen [1]. En France, aucune donnée précise sur la prévalence de cette maladie n'existe, mais on estime que près d'un million de Français présenterait cette maladie.

Dans les pays à revenu faible/intermédiaire, la prévalence de cette maladie serait plus élevée chez les femmes [1]. Cette constatation n'est pas clairement élucidée, mais peut partiellement s'expliquer par des valeurs d'IPS plus faible chez les femmes, notamment d'origine africaine (cf. ci-dessus) [8]. Une autre explication serait un biais de sélection par l'espérance de vie particulièrement faible des hommes à haut risque cardiovasculaire dans ces pays.

En comparant les données entre 2000 et 2010 (figure 2), la prévalence de l'AOMI a beaucoup plus augmenté dans les pays à revenu faible/intermédiaire que dans les pays à haut revenu [11]. Ajustés à l'âge et à la prévalence des facteurs de risque, les régions du monde ayant vu la plus forte progression de l'AOMI sont les pays de l'Asie du Sud-Est et les régions Ouest-Pacifique [11]. Aucune étude simultanée internationale n'a été réalisée, et les comparaisons ont des limites du fait de méthodologies pouvant être différentes. À titre d'exemple, dans 5 études réalisées en Chine, la prévalence de l'AOMI dans la tranche d'âge de 60 à 70 ans varie de $2,5 \%$ à $6,9 \%$ chez l'homme, et de $1,7 \%$ à $10,4 \%$ chez la femme [3]. Cependant de véritables différences peuvent exister, comme en témoigne l'étude EPIDEMCA ayant trouvé une prévalence d'AOMI différente malgré une méthodologie semblable dans 2 pays voisins d'Afrique Centrale (12,2 \% dans la République du Congo vs. 17,4 \% dans la République (entrafricaine), ceci pouvant être expliqué par des données socioéconomiques et démographiques différentes [12].

L'ensemble de ces études fournit une bonne appréhension de l'épidémiologie de l'AOMI dans son ensemble mais ne peut donner des informations sur I'AOMI clinique. D'autres études, menées en Europe et aux États-Unis, ayant notamment recours aux questionnaires, permettent de définir la prévalence de la claudication intermittente à moins de $1 \%$ avant 50 ans et jusqu'à $6 \%$ après 65 ans [13]. À côté de la claudication intermittente typique, des formes atypiques ont été mises en évidence, notamment du fait de leurs localisations, ou de la poursuite de la marche malgré la douleur. La prévalence de ces formes atypiques est encore moins connue. Dans une étude hollandaise, la prévalence de la claudication intermittente typique était de 1,6\%, alors que $5 \%$ présentaient des signes atypiques [14]. Dans l'avenir, il sera indispensable de confirmer ces formes atypiques, parfois intriquées avec d'autres pathologies, par l'IPS ou d'autres examens paracliniques.

Les données concernant l'ischémie critique sont encore plus parcellaires. Aux États-Unis, à partir des demandes de prise en charge des bénéficiaires de Medicare et Medicaid, la prévalence de l'ischémie critique a été estimée à 2,3\% de la 


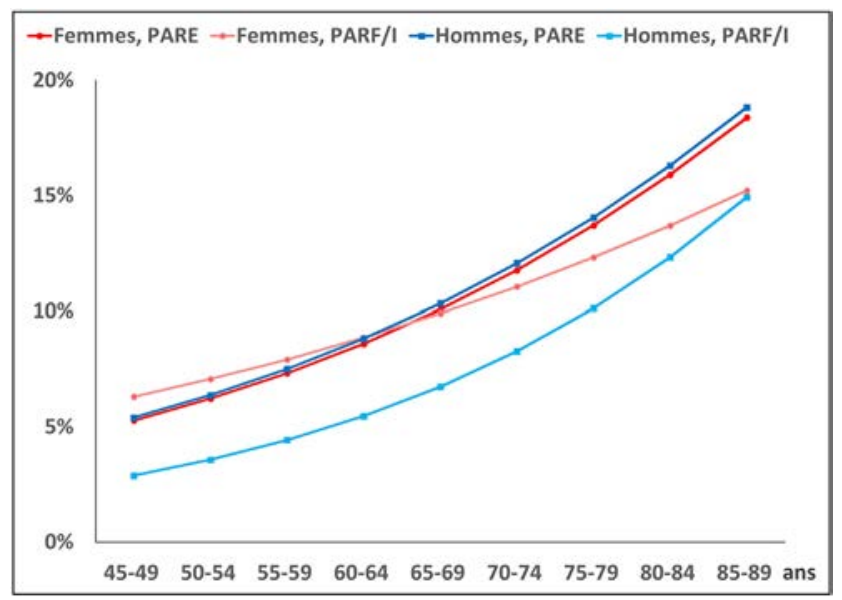

FIgURE 1

Prévalence de l'artériopathie oblitérante des membres inférieurs (AOMI) chez les hommes et les femmes dans les pays à revenu élevé (PARE) et les pays à revenu faible/intermédiaire (PARF/I) - d'après l'étude Global Burden Disease 2010 [1,11]

population américaine de plus de 40 ans [15]. En Suède, celle-ci est estimée à $0,5 \%$ [16].

\section{Incidence}

La prévalence des formes cliniques de l'AOMI étant relativement faible, les études sur l'incidence sont d'autant plus difficiles à mener, avec des marges d'erreur non négligeables. Vers l'âge de 60 ans, l'incidence annuelle de la claudication intermittente a été rapportée à $1 \%$ en Israël et $0,2 \%$ en Islande $[17,18]$. Dans l'étude Framingham, l'incidence annuelle augmente avec l'âge, de 0,4 pour mille hommes âgés de 35-45 ans à 6 pour mille hommes âgés de plus de 65 ans [19]. Concernant l'ischémie critique, son incidence annuelle est estimée à 0,35 \% aux États-Unis [15]. En Angleterre, cette prévalence est estimée nettement plus faible à $0,02 \%$ [20]. Une autre approche est d'étudier les taux d'amputations d'origine non traumatique, même si elles ne sont pas toujours d'origine ischémique. Une étude internationale (Europe, Amérique du Nord et Extrême-Orient) rapporte de grandes disparités avec une incidence annuelle pour une première amputation variant de 2,8 pour 100000 habitants à Madrid à 44 pour 100000 chez les indiens Navajo aux États-Unis chez qui la prévalence du diabète est très forte [21]. L'amputation a été étudiée auprès de 4 millions d'allemands entre 2006 et 2012 : l'incidence de l'amputation majeure est restée stable à 0,04\% [22]. L'incidence annuelle de l'amputation mineure a été de $0,03 \%$. Rapportée à la population allemande, plus de 49000 personnes ont subi une amputation majeure, ce chiffre étant de 32000 pour les amputations mineures. En Hongrie, le taux annuel d'amputation majeure liée à l'AOMI est resté stable entre 2004 et 2012, de l'ordre de 42 pour 10000 habitants [23]. Ce même taux était de 318/10 000 sujets diabétiques.

\section{Les facteurs de risque}

L'A0MI partage, avec les autres maladies athéromateuses, les mêmes facteurs de risque. Cependant, le poids de chaque facteur de risque diffère entre l'AOMI et les autres maladies

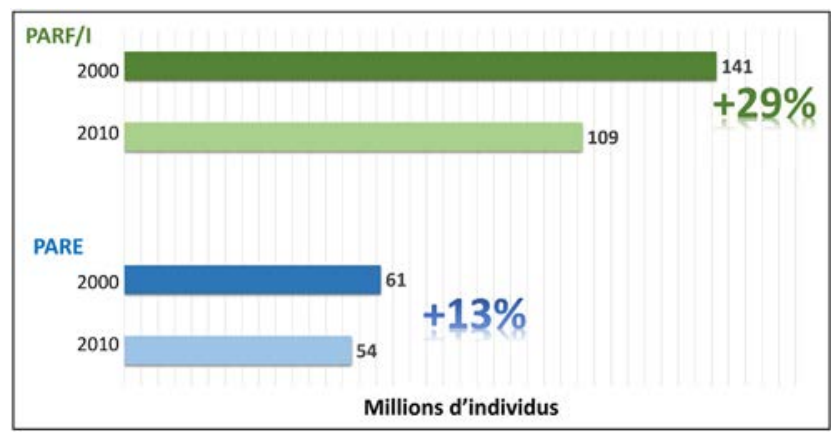

FIGURE 2

Nombre d'individus atteints d'artériopathie oblitérante des membres inférieurs (AOMI) dans le monde dans les pays à revenu élevé (PARE) et ceux à revenu faible/intermédiaire (PARF/I) : évolution entre 2000 et 2010 - d'après l'étude Global Burden Disease [1] 
athéromateuses, et notamment coronaires. Cette maladie est le fruit d'une interaction entre le génotype individuel et l'exposition aux facteurs environnementaux. Les facteurs de risque sont globalement de 4 ordres : les facteurs génétiques, les facteurs de risque traditionnels, les facteurs métaboliques et inflammatoires, et les facteurs psycho-socioéconomiques. Les facteurs les plus étudiés et leur association avec l'AOMI sont présentés sur la figure 3 à travers le projet international The Global Burden Study [1]. Globalement, le contrôle des facteurs de risque traditionnels a un effet favorable tant sur la diminution du risque de survenue de la maladie que son évolution et ses complications.

\section{Tabagisme}

Toutes les études concordent pour trouver que le tabagisme est probablement le facteur le plus puissant pourvoyeur d'AOMI, même si elles diffèrent dans leurs modes d'estimation du tabagisme, souvent qualifié par catégories (jamais, passé, actuel) ou bien par une certaine quantité de tabagisme, par exemple en paquets-années. Globalement, le tabagisme multiplie par 2 à 3 le risque de l'AOMI [2,3]. Dans l'étude française multicentrique COPART, $3 / 4$ des patients hospitalisés pour AOMI étaient ou avaient été fumeurs ; près de $12 \%$ étaient même atteints de bronchite chronique [24].

Selon une étude longitudinale d'hommes travaillant aux ÉtatsUnis, la part contribuable du tabagisme à la présence d'AOMI dans cette population a été estimée à $44 \%$ [25]. Cette part attribuable tient compte de l'intensité de l'association et de la grande fréquence de ce facteur de risque. La diminution du tabagisme dans la population aura probablement une influence dans l'avenir sur l'épidémiologie de cette maladie, avec une préoccupation particulière chez les femmes du fait d'une forte augmentation de tabagisme chez les femmes jeunes. L'arrêt du tabagisme est associé à une diminution progressive du risque
d'A0MI, mais même 20 ans après l'arrêt, un sur-risque persiste [25].

Le risque d'AOMI lié au tabagisme passif est peu étudié. Dans une étude chinoise, les femmes confrontées au tabagisme passif présentent un excès de risque de $+67 \%$ de développer une AOMI, avec un effet-dose significatif tant lié à la durée d'exposition qu'à l'intensité de celle-ci [26].

\section{Diabète}

Le diabète est un facteur puissant et indépendant de survenue d'AOMI, cette association étant plus forte lorsqu'on étudie les formes graves de la maladie, et notamment l'ischémie critique et le risque d'amputation [27-29]. Le risque d'AOMI est globalement multiplié par 2 à 4 , et ce risque augmente avec la durée du diabète. Dans sa forme asymptomatique, l'étude de l'association entre le diabète et un IPS bas est rendue difficile par la présence potentielle de médiacalcose, rendant les artères de chevilles peu ou pas compressibles, pouvant ainsi diminuer la sensibilité de I'IPS à détecter une AOMI [30]. Le diabète étant un facteur de risque moins prévalent dans la population générale, sa part attribuable à la survenue de l'AOMI dans la population est de $14 \%$ [25]. L'explosion épidémiologique du diabète dans nos populations aura bientôt comme conséquence une augmentation de l'artériopathie du diabétique, une forme anatomoclinique particulière de la maladie, avec une atteinte plus distale et moins accessible à une revascularisation efficace, pouvant être par ailleurs associée à une neuropathie et à un plus grand risque d'infection. L'ensemble de ces éléments explique la plus grande incidence d'amputation chez le diabétique, dont le risque est multiplié par 5 comparé à celui d'un artériopathe non diabétique [25].

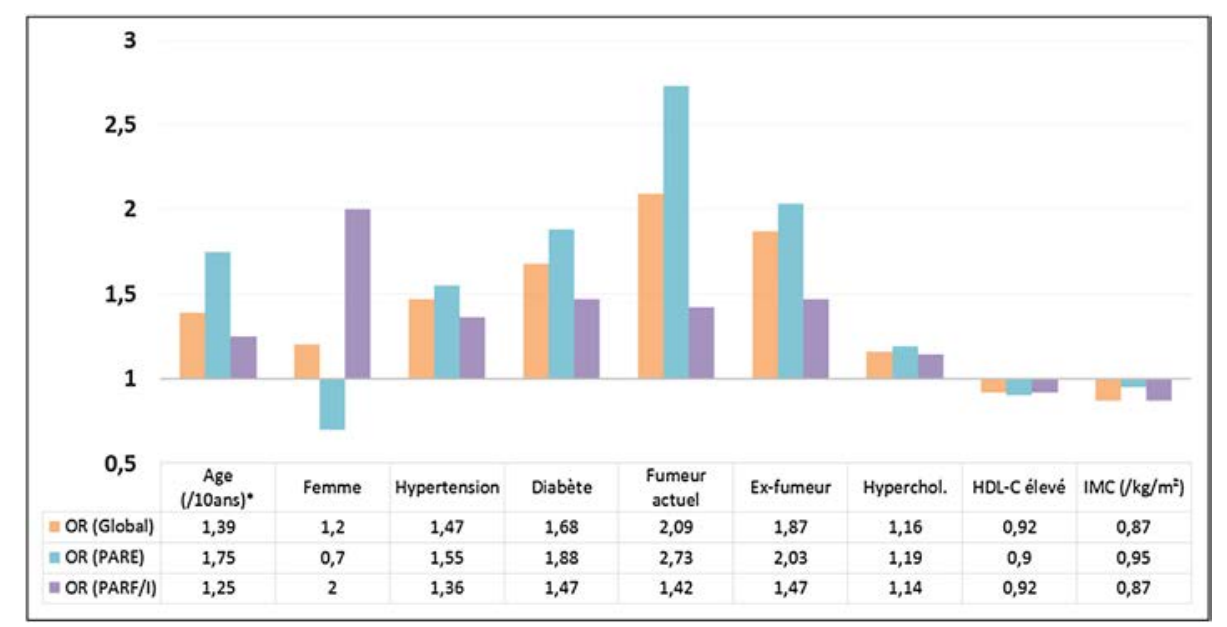

FIGURE 3

Association des principaux facteurs de risque et artériopathie oblitérante des membres inférieurs (A0MI) selon la situation économique des pays - d'après l'étude Global Burden Disease [1] 


\section{Hypertension artérielle (HTA)}

La plupart des études concordent à reconnaître l'HTA comme un facteur de risque indépendant d'AOMI, même si la force de l'association est plus faible, avec un odds ratio variant de 1,5 à 2 [2,3]. Lorsque la pression artérielle est étudiée en variable continue, on retrouve une association significative avec la pression artérielle systolique, et non pas diastolique $[3,28]$. Cependant, ce phénomène peut s'expliquer par la rigidité artérielle souvent importante chez l'artériopathe, avec comme conséquence une baisse de la pression diastolique. Du fait de sa grande prévalence après 60 ans, la part attribuable de l'HTA dans la survenue de l'AOMI est importante, estimée à $17 \%$ dans une étude hollandaise et à près de $41 \%$ dans une étude américaine $[25,28]$.

\section{Dyslipidémies}

L'hypercholestérolémie (totale ou de sa fraction LDL) est un facteur de risque modéré de survenue de l'AOMI, même s'il existe quelques résultats discordants dans la littérature [2]. Sa part attribuable dans l'étude des travailleurs américains est à $17 \%$ [25]. Le meilleur indicateur du risque d'AOMI lié au cholestérol est la fraction cholestérol totale/HDL-c qui tient ainsi compte de l'association divergente des différentes fractions de cholestérol [31,32]. Ainsi, dans l'étude des médecins américains, le ratio cholestérol total/HDL-c était l'indicateur de dyslipidémie le plus puissant pour prédire l'AOMI, avec un risque multiplié par 3,9 pour les patients du quatrième quartile par rapport à ceux du premier quartile [33]. Les statines diminuent la morbi-mortalité cardiovasculaire des patients artériopathes, et réduit également le risque de complications au niveau des membres [34].

Quant au risque lié à l'hypertriglycéridémie, les résultats sont plus contradictoires, mais plusieurs études sont en faveur d'un lien significatif entre cette entité et l'AOMI [2].

\section{Autres facteurs métaboliques et inflammatoires}

L'obésité est un facteur de risque débattu, avec des études aux résultats opposés, ou alors une relation en " U » entre l'obésité et l'AOMI $[2,3]$. Ces résultats contradictoires peuvent en partie être expliqués par la co-existence d'autres maladies chroniques chez les sujets âgés, ainsi que des facteurs confondants ne pouvant pas toujours être parfaitement contrôlés dans les modèles multivariés. Le manque d'exercice, parfois directement limité du fait d'une AOMI, peut être pourvoyeur d'obésité.

L'hyperhomocystéinémie a été longtemps considérée comme un facteur de risque puissant d'AOMI avant que de nouveaux résultats défient les plus anciens [2,3]. La supplémentation en folates n'a jamais fait preuve de son efficacité clinique.

L'insuffisance rénale, même dans sa forme peu sévère, est associée à une augmentation du risque d'AOMI, avec un risque plus net chez le dialysé [2]. La proportion d'insuffisants rénaux en cas d'AOMl sévère (et notamment ischémie critique) est importante. Dans l'étude COPART, $16 \%$ des patients hospitalisés pour AOMI avaient une insuffisance rénale [24].

Plusieurs études ayant recours à des biomarqueurs divers (mais notamment la (RP et le fibrinogène) concordent pour démontrer une association significative entre l'inflammation et la survenue voire la progression de l'AOMI $[35,36]$.

\section{Facteurs génétiques}

Dans les populations multi-éthniques et notamment aux ÉtatsUnis, plusieurs études épidémiologiques se sont attachées à comparer l'épidémiologie de l'AOMI dans les différents groupes ethniques, avec constamment une plus forte prévalence d'AOMI chez les sujets d'origine africaine comparée aux sujets d'origine européenne. À l'inverse, par rapport à ces derniers, les américains d'origine asiatique ou ceux de l'Amérique Latine sont à plus faible risque d'AOMI [3].

Dans plusieurs études américaines réalisées auprès de jumeaux, le déterminisme génétique de l'IPS a été estimé entre $21 \%$ et $48 \%$ [2]. Dans une méta-analyse, certaines variantes de gènes localisées dans la région chromosomique 9p21 étaient liées à la présence d'AOMI [37]. D'autres études n'ont pas confirmé ces résultats [38].

\section{Facteurs psycho-socioéconomiques}

Les facteurs économiques semblent avoir un effet important sur l'épidémiologie de l'AOMI. Nous avons précédemment évoqué les différences constatées selon le niveau de richesse d'un pays. Dans un même pays, un niveau socioéconomique faible est associé à l'AOMI, tant aux États-Unis, en Europe qu'en Afrique $[12,39]$.

Les troubles psychologiques et notamment la dépression [10] sont fréquents chez les artériopathes. Dans une étude, le stress professionnel a été retrouvé associé à un plus fort risque de claudication intermittente [18].

Le lien entre la consommation d'alcool et l'AOMI est très débattu, avec des résultats contradictoires, certaines études retrouvant un effet protecteur d'une faible consommation d'alcool, mais les résultats divergent selon le sexe et selon le pays étudié [3].

\section{Pronostic}

\section{Devenir local}

Le pronostic local du membre inférieur varie selon le stade de la maladie, mais on connaît peu l'histoire naturelle de l'AOMI au stade asymptomatique. Un des moyens de mesurer cette progression, en particulier chez les patients asymptomatiques, est l'évolution de l'IPS au cours du temps. L'apparition d'une ischémie d'effort (claudication intermittente) chez des patients jusqu'à lors asymptomatiques est d'environ $9 \%$ sur un suivi de 5 à 7 ans [40]. Cette progression est plus importante que celle de la population générale, vraisemblablement du fait de la présence de plus de facteurs de risque chez les artériopathes asymptomatiques, et notamment le tabagisme. Les patients asymptomatiques peuvent bien sûr évoluer rapidement vers des formes 


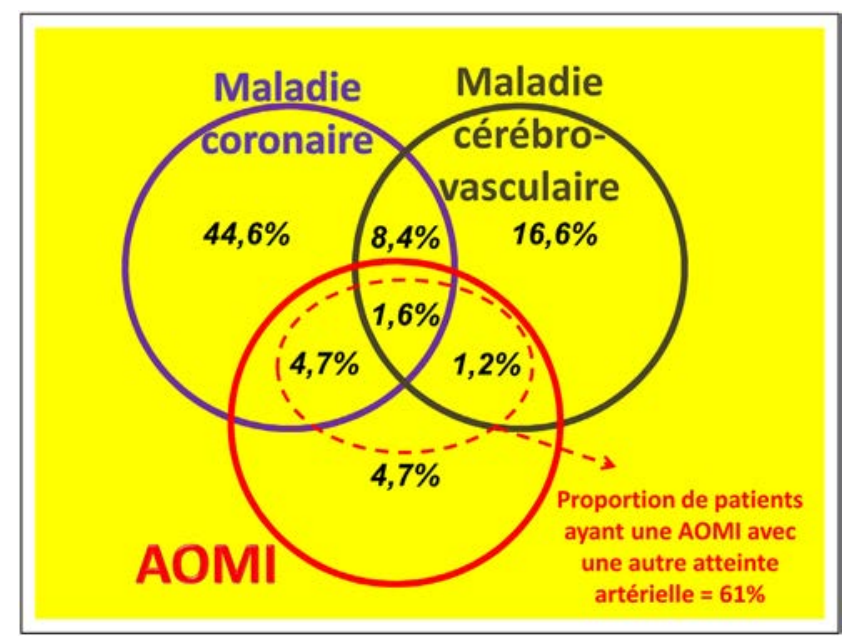

FIGURE 4

Prévalences et associations des principales maladies athéromateuses dans le registre REACH [46]

sévères, du fait de complications aiguës, mais aussi en raison de l'absence de symptômes du fait d'une faible activité physique ou d'une neuropathie diabétique associée. Au stade de la claudication intermittente, on considère que $20 \%$ des patients ont une aggravation de la claudication à 5 ans dont les $2 / 3$ ont une aggravation de la claudication et $1 / 3$ un passage vers l'ischémie critique [41]. Au total, $5 \%$ des patients claudicants sont amputés à 5 ans. Les facteurs de risque de progression de l'AOMI sont l'âge, le tabac, le diabète et la dyslipidémie ainsi que des facteurs inflammatoires [42]. Ces études présentent cependant un biais de survie, puisque l'artériopathie évolutive est également associée à une surmortalité cardiovasculaire [43].

Au stade d'ischémie permanente ou critique, l'évolutivité est difficile à analyser du fait des efforts de revascularisation généralement mis en œuvre. On considère qu'après un an de suivi, $30 \%$ des patients sont amputés, $45 \%$ ne sont pas amputés et $15 \%$ sont décédés [44]. Ces données sont confirmées par celles du registre français COPART montrant une mortalité à un an de $28 \%$ en cas d'ischémie critique [24].

Au total, l'évolutivité locale de l'AOMI est loin d'être inéluctable et suit globalement la sévérité de la maladie locale. Elle se stabilise souvent au stade de claudication intermittente et dépend bien sûr de la thérapeutique médicale et notamment de la prise en charge des facteurs de risque. Au stade d'ischémie critique, le sauvetage du membre est en jeu et le pronostic local est souvent compromis. Globalement, le risque local est moins sévère que le risque général.

\section{Pronostic général}

Il n'est pas étonnant de constater que les patients souffrant d'AOMI ont plus de maladies cardio- ou cérébrovasculaires que la population générale, du fait des facteurs de risque communs à ces maladies et du substratum commun qu'est l'athérosclérose. Le risque d'infarctus du myocarde est multiplié par 2,5 et le risque d'AVC et d'insuffisance cardiaque respectivement par 3,1 et 3,3 [45]. Les registres montrent également une importante prévalence de l'AOMI chez les patients coronariens ou cérébrovasculaires (figure 4) [46].

L'AOMI est une puissant prédicteur de morbi-mortalité cardiovasculaire même après ajustement sur les facteurs de risque cardiovasculaires [4]. Si l'atteinte des artères des membres inférieurs n'est pas directement responsable des manifestations d'autres localisations, elle a une valeur pronostique importante, tant dans sa forme asymptomatique que ses formes cliniques. Plusieurs études épidémiologiques et de suivi de cohortes ont montré que I'IPS variait de façon inversement proportionnelle à la morbi-mortalité cardiovasculaire. C'est particulièrement vrai pour la maladie coronaire et un peu moins pour la pathologie cérébrovasculaire. L'IPS apparaît comme un excellent marqueur du risque cardiovasculaire et de la mortalité cardiovasculaire. Au-dessous de 1,10, plus I'IPS est bas, plus la mortalité cardiovasculaire augmente. Inversement, un IPS trop élevé > 1,40 est également associé à un risque de mortalité élevé, notamment en cas d'AOMI associée.

Les patients atteints d'AOMI ont souvent une atteinte coronaire et une atteinte cérébrovasculaire comme le montre le registre REACH puisque $39 \%$ des patients artériopathes ont une atteinte coronaire, $10 \%$ une atteinte cérébrovasculaire et $13 \%$ ont les deux autres atteintes (figure 4). Ceci se traduit par des causes de décès associées puisque dans les études initiales, 40 à $60 \%$ des causes de décès étaient d'origine coronaire, 10 à $20 \%$ d'origine cérébrovasculaire et $10 \%$ d'autres causes cardiovasculaires [5]. Plus récemment, des études américaines ont montré que la mortalité par cancer était d'importance égale chez ces patients, 
ce qui tient peut-être au fait de l'amélioration du traitement médical chez ces patients, mais aussi de certains facteurs de risque communs tels que le tabagisme et la sédentarité [47]. De façon générale, la mortalité croît avec le nombre de sites atteints ainsi que chez les patients symptomatiques par rapport aux asymptomatiques. À un an, l'incidence des complications cardiovasculaires est d' $1 / 3$ supérieure chez les patients porteurs d'une AOMI, par rapport aux patients cérébrovasculaires ou coronariens [48].

\section{Conclusion}

L'AOMI est un mode d'expression fréquent de l'athérosclérose. Elle est donc associée aux facteurs de risque usuels des maladies athéromateuses, avec une place prépondérante pour le tabagisme, le diabète et l'hypertension artérielle. II s'agit d'une maladie chronique avec une période infraclinique généralement longue, et une expression clinique le plus souvent après 65 ans.
Les pays en voie de développement rattrapent à grand pas le « retard épidémiologique " par rapport aux pays occidentaux, avec aujourd'hui, plus de patients atteints de cette maladie dans les pays en voie de développement que dans les pays riches. Le développement des mesures de prévention cardiovasculaire (lutte contre le tabagisme et la sédentarité) permettent de contrecarrer en partie le vieillissement des populations et l'essor du diabète et des maladies métaboliques, changeant progressivement l'épidémiologie et la présentation de cette maladie au $21^{\mathrm{e}}$ siècle. Le patient touché par cette maladie encourt le double risque d'une aggravation locale de la perfusion des membres inférieurs et celui d'un décès et/ou d'un évènement cardiovasculaire.

Déclaration de liens d'intérêts : V Aboyans : Bayer, Sanofi, BMS/Pfizer, Amgen, Novartis, M-A Sevestre : Bayer, BMS/Pfzer, Aspen et LeoPharma. Les autres auteurs déclarent ne pas avoir de liens d'intérêts..

\section{Références}

[1] Fowkes FG, Rudan D, Rudan I, Aboyans V, Denenberg J0, McDermott MM, et al. Comparison of global estimates of prevalence and risk factors for peripheral artery disease in 2000 and 2010: a systematic review and analysis. Lancet 2013;382:1329-40.

[2] Fowkes FG, Aboyans V, Fowkes FJ, McDermott MM, Sampson UK, Criqui MH. Peripheral artery disease: epidemiology and global perspectives. Nat Rev Cardiol 2017;14:156-70.

[3] Criqui MH, Aboyans V. Epidemiology of peripheral artery disease. Circ Res 2015;116:1509-26.

[4] Criqui MH, Langer RD, Fronek A, Feigelson HS, Klauber MR, McCann T), et al. Mortality over a period of 10 years in patients with peripheral arterial disease. N Engl J Med 1992;326:381-6.

[5] Fowkes FG, Murray GD, Butcher I, Heald CL, Lee RJ, Chambless LE, et al. Ankle-brachial index combined with Framingham Risk Score to predict cardiovascular events and mortality: a meta-analysis. JAMA 2008;300:197-208.

[6] Aboyans V, Criqui MH, Abraham P, Allison MA, Creager MA, Diehm C, et al. Measurement and interpretation of the ankle-brachial index: a scientific statement from the American Heart Association. Circulation 2012;126: 2890-909.

[7] Lijmer JG, Hunink MG, van den Dungen JJ, Loonstra J, Smit AJ. ROC analysis of noninvasive tests for peripheral arterial disease. Ultrasound Med Biol 1996;22:391-8.

[8] Aboyans V, Criqui MH, McClelland RL, Allison MA, McDermott MM, Goff Jr DC, et al. Intrinsic contribution of gender and ethnicity to normal ankle-brachial index values: the Multi-Ethnic Study of Atherosclerosis (MESA). J Vasc Surg 2007;45:319-27.

[9] Aboyans V, Lacroix P, Waruingi W, Bertin F, Pesteil F, Vergnenègre $A$, et al. Traduction française et validation du questionnaire
d'Edimbourg pour le diagnostic de la claudication intermittente. Arch Mal Coeur Vaiss 2000;93:1173-7.

[10] Brostow DP, Petrik ML, Starosta A), Waldo SW. Depression in patients with peripheral arterial disease: a systematic review. Eur J Cardiovasc Nurs 2017;16:181-93.

[11] Sampson UK, Fowkes FG, McDermott MM, Criqui MH, Aboyans V, Norman PE, et al. Global and regional burden of death and disability from peripheral artery disease: 21 world regions, 1990 to 2010. Glob Heart 2014;9:145-58.

[12] Desormais I, Aboyans V, Guerchet $M$, Ndamba-Bandzouzi B, Mbelesso P, Dantoine $T$, et al. Prevalence of peripheral artery disease in the elderly population in urban and rural areas of Central Africa: the EPIDEMCA study. Eur J Prev Cardiol 2015;22:1462-72.

[13] Norgren L, Hiatt WR, Dormandy JA, Nehler MR, Harris KA, Fowkes FG, et al. Inter-Society Consensus for the Management of Peripheral Arterial Disease (TASC II). Eur J Vasc Endovasc Surg 2007;33(Suppl. 1):S1-75.

[14] Stoffers HE, Rinkens PE, Kester AD, Kaiser $V$, Knottnerus JA. The prevalence of asymptomatic and unrecognized peripheral arterial occlusive disease. Int J Epidemiol 1996;25:282-90.

[15] Nehler M, Duval S, Diao L, Annex BH, Hiatt WR, Rogers K, et al. Epidemiology of peripheral arterial disease and critical limb ischemia in an insured national population. J Vasc Surg 2014;60:686-95.

[16] Sigvant B, Wiberg-Hedman K, Bergqvist D, Rolandsson D, Andersson B, Persson E, et al. A population-based study of peripheral arterial disease prevalence with special focus on critical limb ischaemia and sex differences. J Vasc Surg 2007;45:1185-91

[17] Ingolfsson 10, Sigurdsson G, sigvaldason $\mathrm{H}$, Thorgeirsson G, Sigfusson N. A marked decline in the prevalence and incidence of intermittent claudication in Icelandic men 1968-1986: a strong relationship to smoking and serum cholesterol - the Reykjavik Study. Clin Epidemiol 1994;47:1237-43.

[18] Bowlin SJ, Medalie JH, Flocke SA, Zyzanski SJ, Goldbourt U. Epidemiology of intermittent claudication in middle-aged men. Am J Epidemiol 1994;140:418-30.

[19] Kannel WB, McGee DL. Update on some epidemiologic features of intermittent claudication: the Framingham Study. J Am Geriatr Soc 1985:33:13-8

[20] Rothwell PM, Coull AJ, Silver LE, Fairhead JF, Giles MF, Lovelock CE, et al. Population-based study of event-rate, incidence, case fatality, and mortality for all acute vascular events in all arterial territories (Oxford Vascular Study). Lancet 2005;366:1773-83.

[21] Global Lower Extremity Amputation Study Group. Epidemiology of lower extremity amputation in centres in Europe, North America and East Asia. The Global Lower Extremity Amputation Study Group. Br J Surg 2000;87: 328-37.

[22] Heyer K, Debus ES, Mayerhoff L, Augustin M. Prevalence and regional distribution of lower limb amputations from 2006 to 2012 in Germany: a population-based study. Eur J Vasc Endovasc Surg 2015;50(6):761-6.

[23] Kolossvary E, Ferenci T, Kovats T, Kovacs L, Jarai Z, Menyhei G, et al. Trends in major lower limb amputation related to peripheral arterial disease in Hungary: a nationwide study (2004-2012). Eur J Vasc Endovasc Surg 2015;50:78-85

[24] Cambou JP, Aboyans V, Constans J, Lacroix P, Dentans C, Bura A. Characteristics and outcome of patients hospitalised for lower extremity peripheral artery disease in France: the 
COPART Registry. Eur I Vasc Endovasc Surg 2010;39:577-85.

[25] Joosten MM, Pai JK, Bertoia ML, Rimm EB, Spiegelman D, Mittleman MA, et al. Associations between conventional cardiovascular risk factors and risk of peripheral artery disease in men. JAMA 2012;308:1660-7.

[26] He Y, Lam TH, Jiang B, Wang J, Sai X, Fan L, et al. Passive smoking and risk of peripheral arterial disease and ischemic stroke in Chinese women who never smoked. Circulation 2008;118:1535-40.

[27] Murabito JM, D'Agostino RB, Silbershatz $H$, Wilson WF Intermittent claudication. A risk profile from The Framingham Heart Study. Circulation 1997:96:44-9.

[28] Meijer WT, Grobbee DE, Hunink MG, Hofman A, Hoes AW. Determinants of peripheral arterial disease in the elderly: the Rotterdam study. Arch Intern Med 2000;160:2934-8.

[29] Allison MA, Criqui MH, Mcclelland RL, Scott JM, McDermott MM, Liu K, et al. The effect of novel cardiovascular risk factors on the ethnicspecific odds for peripheral arterial disease in the Multi-Ethnic Study of Atherosclerosis (MESA). J Am Coll Cardiol 2006;48:1190-7.

[30] Aboyans V, Ho E, Denenberg J0, Ho LA, Natarajan L, Criqui MH. The association between elevated ankle systolic pressures and peripheral occlusive arterial disease in diabetic and nondiabetic subjects. J Vasc Surg 2008:48:1197-203.

[31] Curb JD, Masaki K, Rodriguez BL, Abbott RD, Burchfiel CM, Chen R, et al. Peripheral artery disease and cardiovascular risk factors in the elderly. The Honolulu Heart Program. Arterioscler Thromb Vasc Biol 1996;16:1495-500.

[32] Ness J, Aronow WS, Ahn C. Risk factors for symptomatic peripheral arterial disease in older persons in an academic hospital-based geriatrics practice. J Am Geriatr Soc 2000;48:312-4.

[33] Ridker PM, Stampfer MJ, Rifai N. Novel risk factors for systemic atherosclerosis: a comparison of C-reactive protein, fibrinogen, homocysteine, lipoprotein(a), and standard cholesterol screening as predictors of peripheral arterial disease. JAMA 2001;285:2481-5.
[34] Kumbhani DJ, Steg PG, Cannon CP, Eagle KA Smith Jr SC, Goto S, et al. Statin therapy and long-term adverse limb outcomes in patients with peripheral artery disease: insights from the REACH registry. Eur Heart J 2014;35:2864-72.

[35] Lowe GD, Fowkes FG, Dawes J, Donnan PT, Lennie SE, Housley E. Blood viscosity, fibrinogen, and activation of coagulation and leukocytes in peripheral arterial disease and the normal population in the Edinburgh Artery Study. Circulation 1993:87:1915-20.

[36] Aboyans V, Criqui MH, Denenberg J0, Knoke JD, Ridker PM, Fronek A. Risk factors for progression of peripheral arterial disease in large and small vessels. Circulation 2006;113:2623-9.

[37] Knowles JW, Assimes TL, Li I, Quertermous T, Cooke JP. Genetic susceptibility to peripheral arterial disease: a dark coroner in vascular biology. Arterioscler Thromb Vasc Biol 2007;27:2068-78.

[38] Wassel CL, Lamina C, Nambi V, Coassin S, Mukamal KJ, Ganesh SK, et al. Genetic determinants of the ankle-brachial index: a meta-analysis of a cardiovascular candidate gene 50K SNP panel in the candidate gene association resource (CARe) consortium. Atherosclerosis 2012;222:138-47

[39] Pande RL, Creager MA. Socioeconomic inequality and peripheral artery disease prevalence in US adults. Circ Cardiovasc Qual Outcomes 2014;7:532-9

[40] Leng GC, Lee AJ, Fowkes FG, Whiteman M, Dunbar J, Housley E, et al. Incidence, natural history and cardiovascular events in symptomatic and asymptomatic peripheral arterial disease in the general population. Int J Epidemiol 1996;25:1172-81.

[41] Sigvant B, Lundin F, Wahlberg E. The risk of disease progression in peripheral arterial disease is higher than expected: a meta-analysis of mortality and disease progression in peripheral arterial disease. Eur J Vasc Endovasc Surg 2016;51:395-403

[42] Kennedy M, Solomon C, Manolio TA, Criqui MH, Newman AB, Polak JF, et al. Risk factors for declining ankle-brachial index in men and women 65 years or older: the Cardiovascular Health Study. Arch Intern Med 2005;165:1896-902.
[43] Criqui MH, Ninomiya JK, Wingard DL, Ji M, Fronek A. Progression of peripheral arterial disease predicts cardiovascular disease morbidity and mortality. J Am Coll Cardiol 2008;52:1736-42.

[44] Hirsch AT, Haskal Z], Hertzer NR, Bakal CW, Creager MA, Halperin JL, et al. ACC/AHA 2005 guidelines for the management of patients with peripheral arterial disease (lower extremity, renal, mesenteric, and abdominal aortic): executive summary a collaborative report from the American Association for Vascular Surgery/Society for Vascular Surgery, Society for Cardiovascular Angiography and Interventions, Society for Vascular Medicine and Biology, Society of Interventional Radiology, and the ACC/AHA Task Force on Practice Guidelines (Writing Committee to Develop Guidelines for the Management of Patients With Peripheral Arterial Disease) endorsed by the American Association of Cardiovascular and Pulmonary Rehabilitation; National Heart, Lung, and Blood Institute; Society for Vascular Nursing; TransAtlantic Inter-Society Consensus; and Vascular Disease Foundation. I Am Coll Cardiol 2006:47:1239312.

[45] Newman AB, Siscovick DS, Manolio TA, Polak J. Fried LP, Borhani NO, et al. Ankle-arm index as a marker of atherosclerosis in the Cardiovascular Health Study. Cardiovascular Heart Study (CHS) Collaborative Research Group. Circulation 1993:88:837-45.

[46] Bhatt DL, Steg PG, Ohman EM, Hirsch AT, Ikeda Y, Mas JL, et al. International prevalence, recognition, and treatment of cardiovascular risk factors in outpatients with atherothrombosis. JAMA 2006;295:180-9.

[47] Vidula $H$, Tian L, Liu K, Criqui MH, Ferrucci L, Pearce WH, et al. Biomarkers of inflammation and thrombosis as predictors of near-term mortality in patients with peripheral arterial disease: a cohort study. Ann Intern Med 2008; 148:85-93.

[48] Steg PG, Bhatt DL, Wilson PW, D'Agostino Sr R, Ohman EM, Röther J, et al. One-year cardiovascular event rates in outpatients with atherothrombosis. JAMA 2007;297:1197-206. 